\title{
Forming Carbon-Nanotube Clusters Using Dielectrophoresis for Respiratory Sensing Applications
}

\author{
Shu-Wei Tung, Yi-Chung Lan and Yao-Joe Yang* \\ Department of Mechanical Engineering, National Taiwan University, \\ No. 1, Sec. 4, Roosevelt Rd., Taipei 10617, Taiwan
}

(Received June 10, 2013; accepted August 21, 2013)

Key words: respiratory sensor, carbon nanotubes, dielectrophoretic assembly

In this paper, we present the development of a carbon-nanotube (CNT) resistive respiratory sensor for detecting and monitoring human breath. The sensing element of the device is realized by forming a cluster of multi-walled CNTs (MWCNTs) on two planar gold-sensing electrodes using the dielectrophoresis (DEP) technique. A DEP electrode probe was proposed for creating the CNT cluster. The electrode pair on a silicon substrate was patterned using a standard lift-off technique. An air-controlled polymethylmethacrylate (PMMA) heating chamber was designed and implemented to accelerate the DEP process as well as achieve a uniformly shaped CNT cluster. While the respiratory gas flows across the MWCNT cluster, the vapor molecules condensing on the junctions between neighboring MWCNTs cause variations in the resistance of the MWCNT cluster. Preliminary measurement results show that the proposed sensor has an excellent sensitivity and a rapid response with a high signal-to-noise ratio, and is suitable for detecting and monitoring human breath under various conditions.

\section{Introduction}

Assessing the respiratory state of patients under critical conditions is one of the essential objectives in health care. Respiration characteristics provide crucial indications of the psychological and physical conditions of a patient. In addition, continuously measuring the respiration of a patient is essential in critical or high-risk situations. Recent progress in the study of breath analysis for clinical diagnosis has been stimulated by the improvement of rapid noninvasive measurement instruments. Various approaches for measuring breath have been proposed recently. ${ }^{(1-4)}$ By using pressure sensors, the pressure change induced by the exhaled gas flow can be detected. In addition, humidity sensors with short response times can be used to measure breath dynamics. Also, the temperature variation occurring near the nose during breathing can be measured using temperature sensors. However, breath measurements taken using *Corresponding author: e-mail: yjy@mems.me.ntu.edu.tw 
pressure- or temperature-sensing techniques might be disturbed by the ambient flow or by temperature fluctuations. In addition, the typical response time of commercially available humidity sensors (10 s) is longer than the duration of an exhalation (3 s). ${ }^{(5)}$

Carbon nanotubes (CNTs), which have attracted considerable research interest for developing various applications during the past decade, are chiral molecules consisting of either a single outer wall (single-walled CNTs or SWCNTs), ${ }^{(6)}$ or multiple nestled walls (multi-walled CNTs or MWCNTs). Because of their unique electrical, physical, mechanical, and chemical properties, CNTs can be used as sensing materials that offer excellent sensitivity and rapid response times. In addition, the high surface area-to-volume ratio of the CNTs is well suited for physical adsorption or chemical interaction. ${ }^{(7-9)}$ Compared with traditional sensing materials, such as semiconducting metal oxides, CNT-based sensing materials have various advantages, such as high sensitivity, room-temperature operability, and compatibility with miniaturization and array designs. ${ }^{(10)}$ A microfabricated respiratory sensor, which takes advantage of the tip effect of the MWCNTs, has been proposed.(11) Gelperin et al. presented a new sensor technology for electronic olfaction that might be employed to develop sensor chips based on DNA-decorated SWCNTs. ${ }^{(12)}$ Kuzmych et al. proposed the detection of exhaled gas using $\mathrm{NO} / \mathrm{NO}_{2}$ oxidation and CNT-based fieldeffect transistor devices.(13) In addition, Hou et al. demonstrated a microdevice that employs CNT-based composite electrodes for monitoring human breath dynamics. ${ }^{(14)}$

In this paper, we propose a novel approach for realizing a respiratory sensor by employing a cluster of MWCNTs on two planar electrodes using a dielectrophoresis (DEP) technique. ${ }^{(15,16)}$ A DEP electrode platform and an air-controlled polymethylmethacrylate (PMMA) heating device are employed to form an MWCNT cluster on a sensing electrode pair. The proposed respiratory sensor exhibits an excellent response and recovery performance to the exhaled flow with a sampling rate of three times per second. The proposed sensor is suitable for detecting and monitoring human breath under various conditions.

\section{Design and Principle}

DEP is a well-known and effective method to align CNTs on electrodes. As an external AC electric field is applied, CNTs in a dispersed solution tend to align with the direction of the external field through the action of the DEP force. ${ }^{(17)}$ The operational principle of assembling the MWCNT cluster by using DEP is shown in Fig. 1(a). A sharp

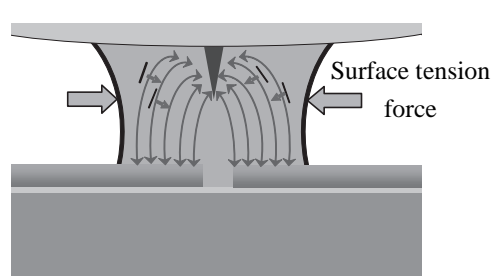

(a)

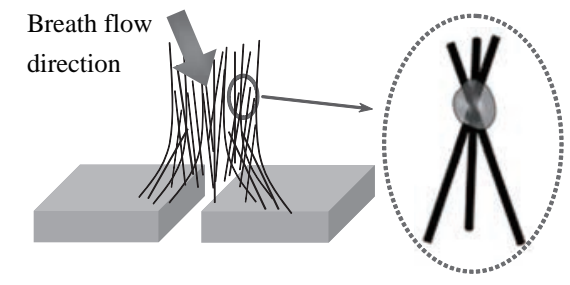

(b)

Fig. 1. (a) Operational principle of assembling an MWCNT cluster. (b) Operational principle of proposed MWCNT cluster respiratory sensor. 
metal tip serves as the DEP top electrode. Two planar metal electrodes serve as the DEP bottom electrode. During the DEP process, the two planar electrodes (i.e., the bottom electrode) are shorted. A liquid droplet is placed between the DEP top electrode and the DEP bottom electrode. When the electric field is applied between the DEP top and bottom electrodes, the MWCNTs rotate to align with the external field direction through the DEP force and then MWCNTs are attracted to the region of high field intensity. ${ }^{(18,19)}$ As the solvent (i.e., ethanol solution) evaporates, the surface tension acts as a binding force to bind the MWCNTs, which are aligned with the external field direction, and forms an MWCNT cluster.

Figure 1(b) shows the operational principle of the proposed respiratory sensor. During the breath measurement, the two bottom planar electrodes are connected to a multimeter. The respiratory gas flows across the MWCNT cluster, and the condensation of breath vapor begins at the junctions between neighboring MWCNTs. ${ }^{(20)}$ The accumulation of the adsorbed vapor molecules subsequently gives rise to an increase in the resistance of the MWCNT cluster. During inspiration, the vapor molecules evaporate rapidly, and the resistance of the MWCNT cluster decreases to the original level.

\section{Device Fabrication}

\subsection{DEP top electrode}

Figure 2 shows the schematic and picture of the DEP electrode for forming the CNT cluster using the DEP effect. The DEP electrode consists of a tungsten tip, a PMMA arm, and a hemispherical polydimethlysiloxane (PDMS). The hemispherical PDMS surface facilitates the control of the shape and position of the MWCNT cluster during the DEP process as the solvent evaporated.

The PDMS layer was fabricated using the soft lithographic process, which is briefly described as follows: the PDMS prepolymer and the curing agent (Sylgard 184A and 184B, Dow Corning) were mixed at a 10:1 ratio. After stirring thoroughly and degassing in a vacuum chamber, the prepared PDMS mixture was poured onto a hemispherical PMMA master. After curing at $90{ }^{\circ} \mathrm{C}$ for $60 \mathrm{~min}$, the cured PDMS layer was peeled from the master substrate.

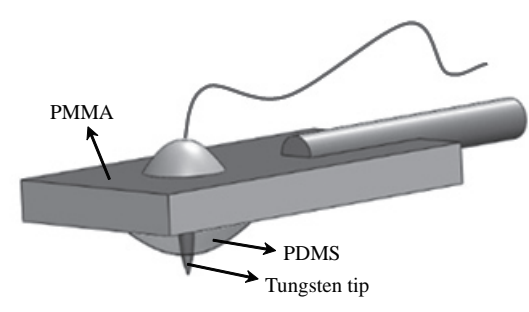

(a)

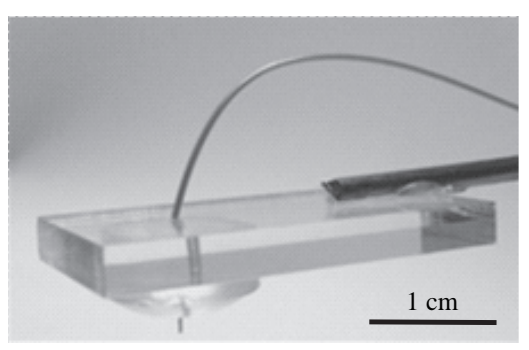

(b)

Fig. 2. (a) Schematic of DEP top electrode and (b) picture of electrode. 


\subsection{Air-controlled heating chamber}

Figure 3 shows the air-controlled PMMA heating chamber for uniform heating during the DEP process. This uniform heating chamber was essential for accelerating the evaporation of the solvent of the CNT solution. The heating chamber was made of PMMA using laser machining. The hot air entered the device with four heat-resistant silicone tubes attached to the input holes. Subsequently, the uniform hot air left the device through four 2 -cm-long and $500-\mu \mathrm{m}$-wide apertures. The mean temperature measured in the center of the chamber was approximately $65^{\circ} \mathrm{C}$.

\subsection{Sensing electrode pair}

Figure 4 shows the fabrication process of the sensing electrode pair, namely, the DEP bottom electrode. As shown in Fig. 4(a), the silicon nitride (3000 $\AA$ ) was deposited on the silicon wafer as the insulation layer by low-pressure chemical vapor deposition (LPCVD). By using E-beam evaporation, chromium (30 nm) and gold (500 nm) were deposited on the insulation layer [Fig. 4(b)]. The chromium film served as an adhesion layer. Then, the pattern of the sensing electrode pair was transferred [Figs. 4(c) and 4(d)]. The sensing electrode pair was formed by using a solution mixed with iodine, potassium iodide, and deionized water (5:10:85) [Fig. 4(e)]. Finally, the residual

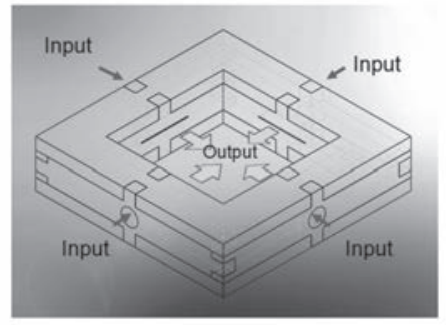

(a)

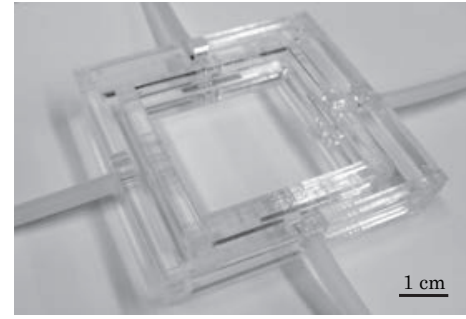

(b)

Fig. 3. Air-controlled PMMA heating chamber: (a) schematic and (b) picture of fabricated chamber.

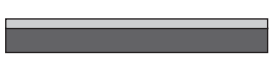

(a)

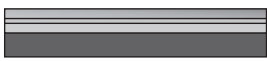

(b)

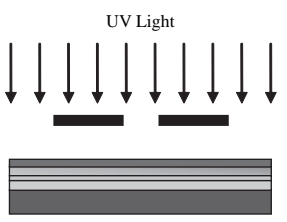

(c)

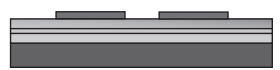

(d)

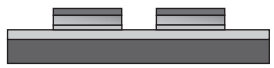

(e)

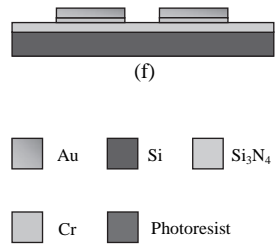

Fig. 4. Fabrication of DEP bottom electrodes (sensing electrode pair). 
photoresist was removed [Fig. 4(f)]. A schematic model and a picture of the fabricated sensing electrode pair are shown in Fig. 5.

\subsection{Forming an MWCNT cluster}

The process of creating an MWCNT cluster can be divided into three steps: (1) dipping the DEP top electrode in the MWCNT solution; (2) placing the DEP top electrode above the center of the bottom electrode pair, which formed the meniscus of a liquid column; and (3) applying the DEP process to form the MWCNT cluster.

Figure 6 shows the process of forming an MWCNT cluster on the bottom planar electrodes (sensing electrodes). Before the DEP process, both of the bottom electrodes were connected and served as a bottom electrode for the DEP process. Regarding the preparation of the MWCNT solution, $100 \mathrm{mg}$ of acid-treated MWCNTs (C tube-009, Golden Innovation Business Co., Ltd.) were suspended in $10 \mathrm{ml}$ of distilled water containing 50 wt.\% ethanol under ultrasonic agitation. The DEP top electrode (Fig. 2) was dipped into the solution mixed with the MWCNTs. Then, the wetted DEP electrode [Fig. 6(a)] was placed above the gap between two bottom DEP electrodes (the sensing electrodes). A meniscus of a liquid column was formed [Fig. 6(b)]. A peak-to-peak AC voltage of $80 \mathrm{~V}$ at a frequency of $1 \mathrm{MHz}$ was applied to the DEP process. The details of the DEP process parameters are listed in Table 1.

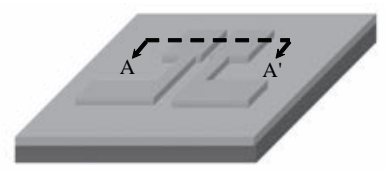

(a)

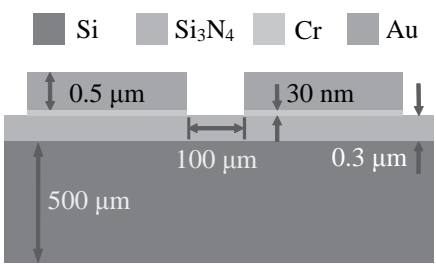

(b)

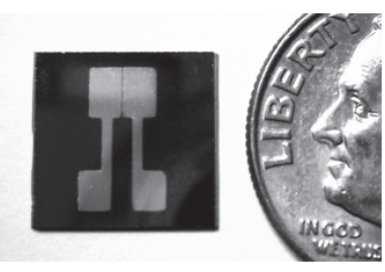

(c)

Fig. 5. (a) 3D model, (b) schematic cross section (not to scale) of sensing electrode pair, and (c) picture of sensing electrodes.

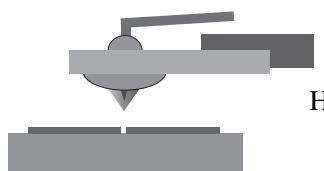

(a)

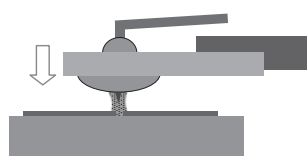

(b)

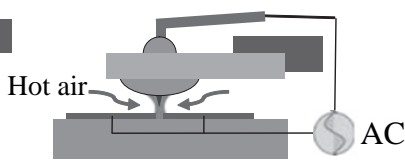

(c)

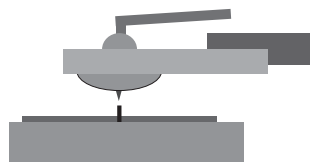

(d)

Fig. 6. Schematic of assembling an MWCNT cluster for the respiratory sensor. 
Table 1

Parameters of forming an MWCNT cluster using DEP.

\begin{tabular}{lc}
\hline Parameters & Definitions/Values \\
\hline DEP electrode & Tungsten tip \\
Sensing electrode pair & Aurum \\
Sensing electrode pair gap & $100 \mu \mathrm{m}$ \\
Voltage (peak to peak) & $80 \mathrm{~V}$ (sinusoidal) \\
Frequency & $1 \mathrm{MHz}$ \\
Experimental time & $15-20 \mathrm{~min}$ \\
\hline
\end{tabular}

Note that the minimum weight percentage of CNT has to be greater than $0.5 \%$ in order to successfully form a cluster. However, it will be extremely difficult to fully disperse CNTs in the solvent if the weight percentage is greater than $2 \%$. In this work, we used 1 wt.\% CNT solution. In addition, the volume of the CNT solution droplet for the DEP process was about $0.004 \mathrm{ml}$. The distance between the top and bottom DEP electrodes is about $200 \mu \mathrm{m}$.

To achieve a uniform shape and the rapid growth of the MWCNT cluster during the DEP process, a heating chamber was employed [Fig. 6(c)]. After the ethanol solution evaporated, an MWCNT cluster was formed [Fig. 6(d)] because of the binding force of the surface tension. The base of the cluster spanned the gap between the two sensing electrodes, enabling the sensing electrodes to detect the electrical properties of the MWCNT cluster. The SEM images of an MWCNT cluster are shown in Fig. 7. The MWCNT cluster is approximately $200 \mu \mathrm{m}$ in height and $100 \mu \mathrm{m}$ in diameter.

\section{Measurement and Discussion}

A schematic diagram of the experiment for measuring the breath curves is shown in Fig. 8(a). The proposed sensor was placed $15 \mathrm{~cm}$ below a volunteer's nostrils. The sensing electrodes were connected to a digital multimeter with two probes. A GPIB ${ }^{\circledR}$ interface was used to transmit the measurement results to a LabVIEW program on a computer for further analysis. The sampling rate in the experiment was three times per second. Figure 8(b) shows the measured transient resistance variations according to normal breath, deep breath, and the air flows of a fan and an air-conditioner. The period cycle of the normal breath is defined as the difference between every two adjacent peaks. The breath behaviors were observed by measuring the resistance of the MWCNT cluster. The rising curve indicates that the volunteer was exhaling. The declining curve indicates that the volunteer was inhaling. Furthermore, the measurement results also revealed that the typical ambient noise sources, such as the airflows from a fan or an air-conditioner, were nominal compared with the breath signals, which indicates that the respiratory sensor exhibits the ability of avoiding the interference of surrounding flow.

Figure 9(a) shows the measured breath characteristics of four volunteers. Each volunteer was tested for three breath cycles for approximately $20 \mathrm{~s}$. The R-t curves show the differences in the breath patterns of the volunteers. In addition, Fig. 9(b) shows the results of a volunteer's breath patterns before and after exercise. Obviously, the volunteer who 

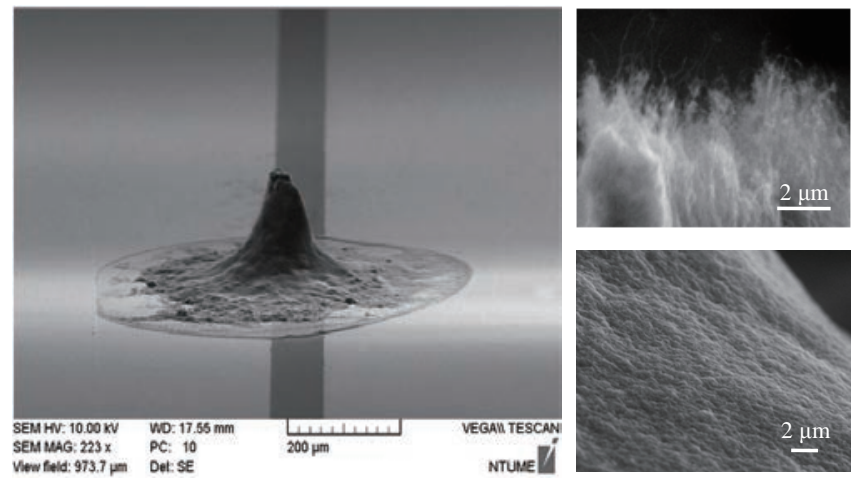

Fig. 7. SEM images of an assembled MWCNT cluster. The top-right picture is the closer view at the top of the CNT cluster, and the bottom-right figure is the closer view at the base of the CNT cluster.

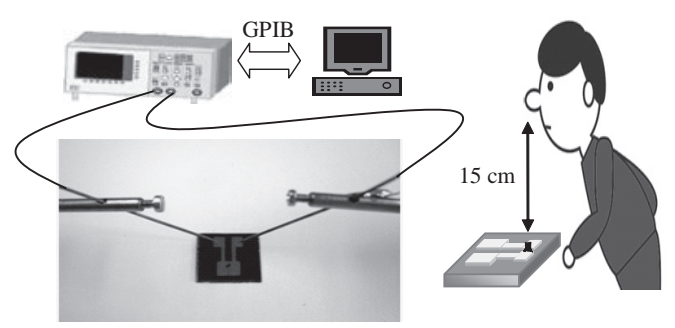

(a)

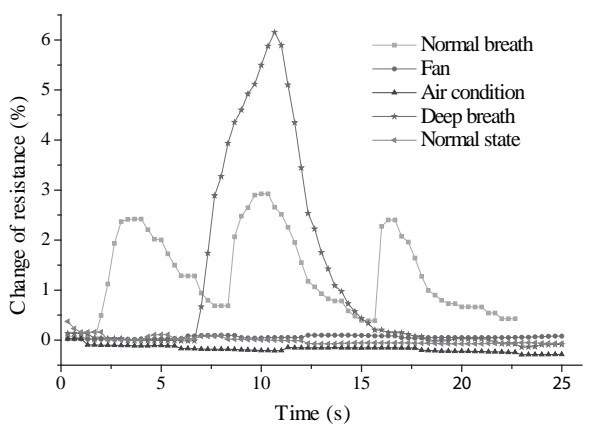

(b)

Fig. 8. (a) Schematic and picture of experimental setup for measuring the breath curve and (b) measured results of breath and typical ambient noises by using the sensor.

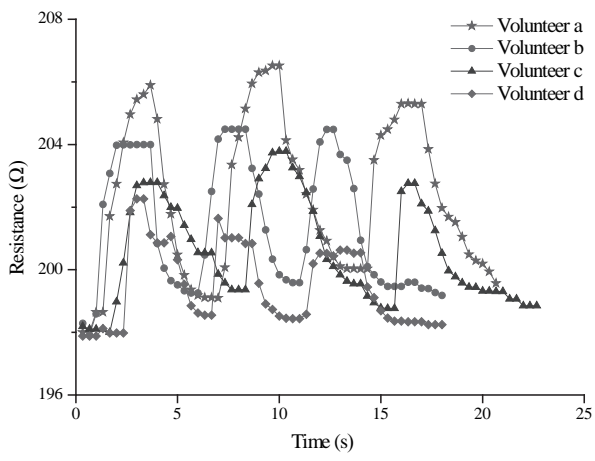

(a)

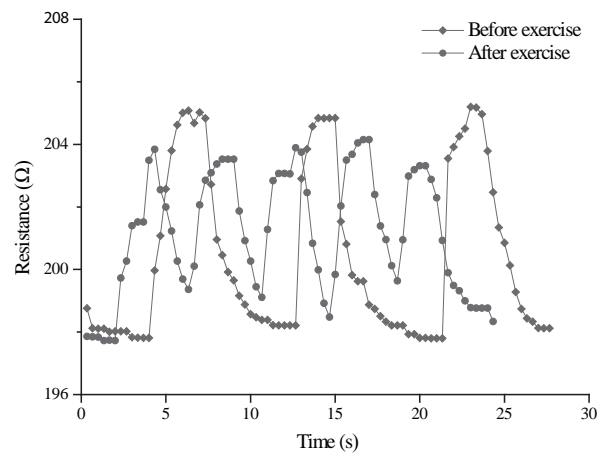

(b)

Fig. 9. (a) Measured breath results of four different volunteers and (b) measured breath results of a volunteer with different respiratory frequencies. 
just finished exercise gave a breath of higher frequency. These experimental results show that the proposed sensor is suitable for detecting and monitoring the human breath under various conditions, and can effectively distinguish between different breath behaviors.

\section{Conclusions}

In this paper, we present a novel resistive respiratory sensor realized by forming a cluster of CNTs on two planar electrodes using a DEP technique. While the respiratory gas flows across the MWCNT cluster, the resistance of the cluster increases because of the condensation of the vapor molecules on the junctions between neighboring MWCNTs. By using an air-controlled PMMA heating chamber during the DEP process, the shape of the MWCNT cluster can be effectively controlled. The experimental results show that the proposed sensor is not only suited for detecting and monitoring human breath but also has excellent sensitivity, a rapid response, and a high signal-to-noise ratio.

\section{Acknowledgements}

This work was partially sponsored by the National Science Council of Taiwan and Foxconn Inc.

\section{References}

1 S. Brady, D. Diamond and K. T. Lau: Sens. Actuators, A 119 (2005) 398.

2 N. Andre, S. Druart, P. Gerard, R. Pampin, L. Moreno-Hagelsieb, T. Kezai, L. A. Francis, D. Flandre and J. P. Raskin: IEEE Sens. J. 10 (2010) 178.

3 A. Tetelin, V. Pouget, J. L. Lachaud and C. Pellet: IEEE Trans. Instrum. Meas. 53 (2004) 1262.

4 A. K. Kalkan, H. D. Li, C. J. O’Brien and S. J. Fonash: IEEE Electron Device Lett. 25 (2004) 526.

5 A. F. P. van Putten, M. J. A. M. van Putten, M. H. P. M. van Putten and P. F. A. M. van Putten: IEEE Sens. J. 2 (2002) 636.

6 S. Iijima and T. Ichihashi: Nature 363 (1993) 603.

7 S. Iijima: Nature 354 (1991) 56.

8 M. S. Dresselhaus: Nature 358 (1992) 195.

9 E. S. Snow, F. K. Perkins, E. J. Houser, S. C. Badescu and T. L. Reinecke: Science 37 (2005) 1942.

10 N. Sinha, J. Z. Ma and J. T. W. Yeow: J. Nanosci. Nanotechnol. 6 (2006) 573.

11 X. H. Chen, Y. Y. Wang, Y. H. Wang, Z. Y. Hou, D. Xu, Z. Yang and Y. F. Zhang: Sens. Actuators, A 158 (2010) 328.

12 A. Gelperin and A. T. C. Johnson: J. Breath Res. 2 (2008) 037015.

13 O. Kuzmych, B. L. Allen and A. Star: Nanotechnology 18 (2007) 375502.

14 Z. Hou, B. C. Cai and D. Xu: Appl. Phys. Lett. 89 (2006) 053105.

15 H. A. Pohl: J. Appl. Phys. 22 (1951) 869.

16 H. A. Pohl: J. Appl. Phys. 29 (1958) 1182.

17 R. Krupke, F. Hennrich, H. von Lohneysen and M. M. Kappes: Science 301 (2003) 344.

18 N. Peng, Q. Zhang, J. Li and N. Liu: J. Appl. Phys. 100 (2006) 024309.

19 D. D. Xu, A. Subramanian, L. X. Dong and B. J. Nelson: IEEE Trans. Nanotechnol. 8 (2009) 449.

20 J. T. W. Yeow and J. P. M. She: Nanotechnology 17 (2006) 5441. 